\title{
Bifurcation Onset Delay in Magnetic Bearing Systems with Auxiliary Bearing and Time Varying Stiffness
}

\author{
M. R. Ghazavi ${ }^{1} \&$ Q. Sun $^{2}$ \\ ${ }^{1}$ Mechanical Engineering Department, Tarbiat Modares University, Tehran, Islamic Republic of Iran \\ ${ }^{2}$ Department of Mechanical and Manufacturing Engineering, University of Calgary, Alberta, Canada \\ Correspondence: M. R. Ghazavi, Mechanical Engineering Department, Tarbiat Modares University, P.O. \\ Box14115-111, Tehran, Islamic Republic of Iran. E-mail: ghazavim@modares.ac.ir
}

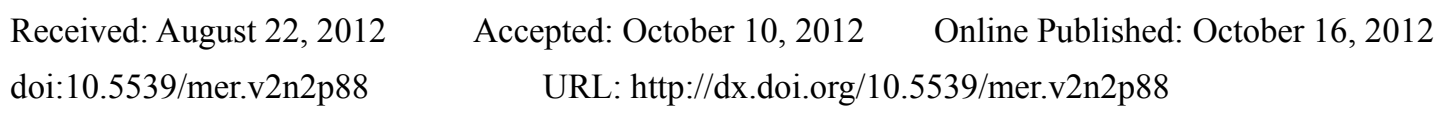

\begin{abstract}
Auxiliary bearings are used in magnetic bearing systemsto protect bearings from damage. These bearings are in contact with rotor temporarily. This contact associated with intermittent contact forces which change the system dynamic behavior. These include vibration instability and thermal stresses. The system is simulated to clarify the role of two different control methods in synchronous and asynchronous responses. This is carried out using linear PD controller and time varying stiffness. Rotor whirl orbit and power spectrum in magneticbearing with time varying stiffness reveals better behavior in some cases. Rotational speed range with synchronous response increases. A delay in the onset of bifurcation depicts an improved rotor dynamic performance.
\end{abstract}

Keywords: magnetic bearings, auxiliary bearings, bifurcation, time varying stiffness

\section{Introduction}

Auxiliary bearings are integral feature of magnetic bearing systems. They are used to protect magnetic bearing specifically during operation. Because of its importance many researches were carried out on this subject especially in recent years. There are two different relative motions for rotor in auxiliary bearing. Contact mode when rotor is in contact with the auxiliary bearing. Contact free mode when the rotor is not in contact.During contact time significant normal and tangential contact forces may impose to rotor. This result load sharing between magnetic bearings and auxiliary bearings. This would affect system dynamic response significantly.

The focus of research by several authors was dynamics of rotor drop onto auxiliary bearings (Zeng, 2002). They obtained transient responses of AMB rotor during rotor drop in different cases. A number of researchers have neglected magnetic bearing force (Wang, 1998). They studied steady state analysis of the dynamic behavior of interactions of the landing rotor with its auxiliary bearings. They have stated that a possible loss of the AMB function, and subsequent interactionsthrough a clearance with the auxiliary bearingof a magnetically supported rotor, lead to a highly nonlinearevent. However some authors considered magnetic force and also rotor flexibility (Jang \& Chen, 2001). The effect of rotor imbalance stiffness on bifurcations response was investigated in their research. But they have neglected geometriccoupling effects in their investigation. They have used linear PD controller in their work. But they have neglected geometric coupling effects in their investigation. However geometric coupling between the two orthogonal axes of motion is an important cause of nonlinearity especially when large rotor displacement is involved. Inayat-Hussain $(2010,2011)$ has developed a model for rigid rotor supported by auxiliary bearings. He considered geometric coupling in his model. He investigated the effect of auxiliary bearing stiffness and friction and rotor imbalance on rotor response. He concluded that imbalance is more significant among thoseparameters. He has used linear PD controller in his work. Cade, Iain et al. carried out an active auxiliary bearing design to reduce contact force (2008). This bearing induces a desirable rotor orbit during and after a contact event. Therefore the dynamic behavior of rotor in clearance circle would be improved. They stated that the contact forces may lead to localized thermal stresses. They also stated that the non-linear nature of contact mechanics due to the auxiliary bearing may cause rotor instability after a contact event. Ghazavi and Sun have used time varying stiffness in magnetic bearing simulation, but they have not considered auxiliary bearings (2011).

In present research a rigid rotor response in magnetic bearing supported by auxiliary bearings are investigated. 
Non linear rotor responses were investigated for a range of speeds. Periodic quasi periodic and chaotic responses are depicted for different speeds. Time varying stiffness is used in this investigation. These induce a better rotor whirl orbit before contact event . The rotor response is compared with that of linear PD controller.

\section{Governing Equations}

Magnetic forces are provided by two sets of electro-magnets. These forces are used to position the rotor.

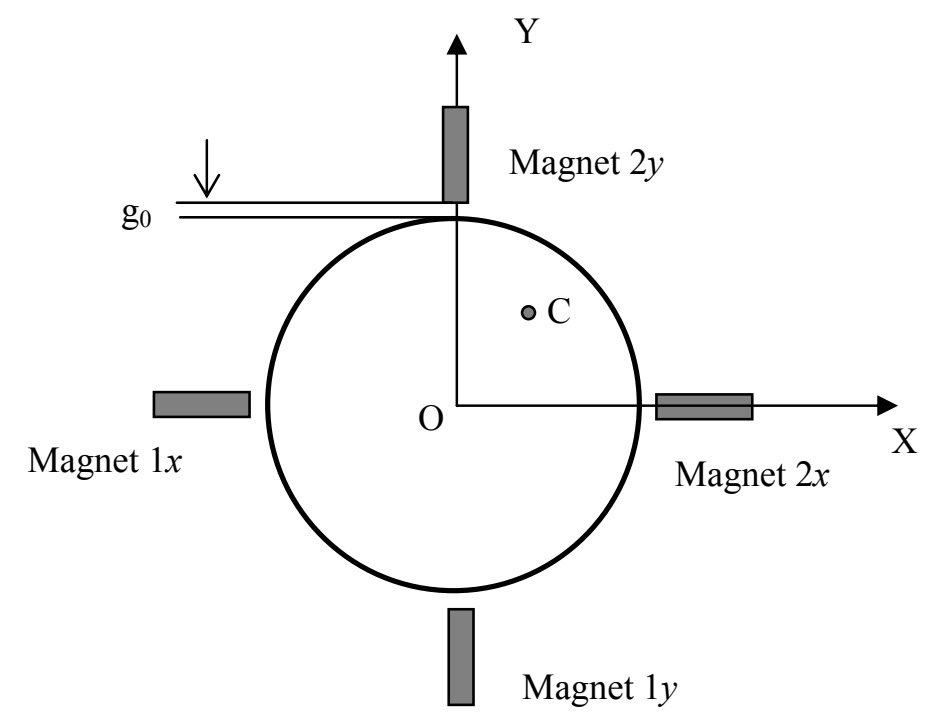

Figure 1. A magnetic bearing and rotor (Ghazavi \& Sun)

A pair of magnets is used in $x$-direction. The $y$-direction equations can be arrived by substituting subscript $\mathrm{x}$ with $y$. Magnetic bearing force for each magnet is calculated using the following equations.

$$
\begin{aligned}
& F_{1 x}=K\left(\frac{i_{b}-i_{x}}{g_{0}+x}\right)^{2} \\
& F_{2 x}=K\left(\frac{i_{b}+i_{x}}{g_{0}-x}\right)^{2}
\end{aligned}
$$

To control the bearing force, the controller generates commands that determine the current flowing through the bearing coils. A common control strategy is proportional-derivative (PD):

$$
i_{x}=-\left(K_{p} x+K_{d} \dot{x}\right)
$$

Where $K_{p}$ and $K_{d}$ are controller proportional and derivative gain constants respectively. We use the same gain values for current command in $y$ direction for a reason that is obvious later, that is, $i_{y}=-\left(K_{p} y+K_{d} \dot{y}\right)$.

This controller is physically similar to linear spring and damper elements in mechanical systems. Since proportional gain acts as stiffness coefficient replacing it with the following terms called time varying stiffness.

$$
K_{p}=P_{1}+P_{2} \cos \omega t
$$




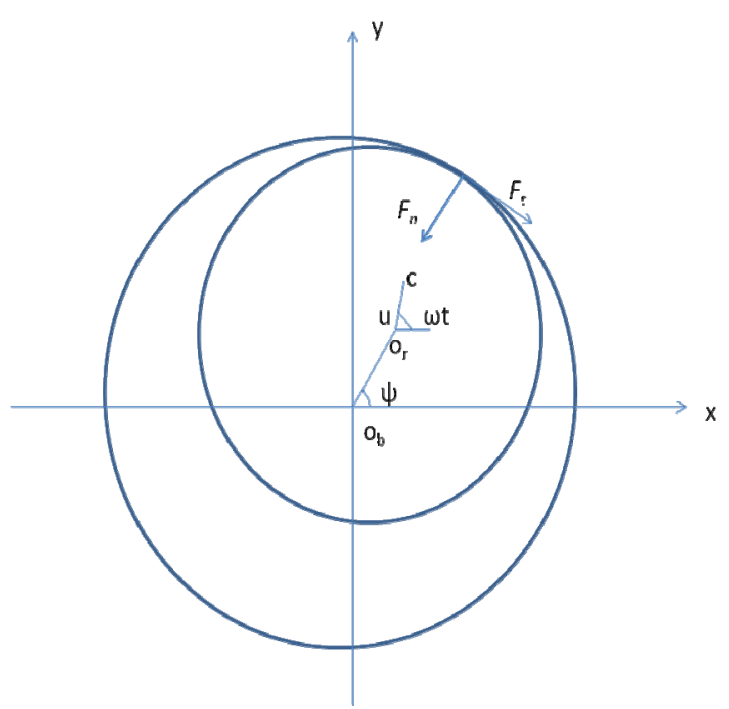

Figure 2. Auxiliary bearing contact forces

Considering geometric coupling the total magnetic forces in $x$ and $y$ directions are:

$$
\begin{gathered}
F_{x}=F_{2 x}-F_{1 x}+\alpha\left(\frac{x}{g_{0}}\right)\left(F_{1 y}+F_{2 y}\right) \\
F_{y}=F_{2 y}-F_{1 y}+\alpha\left(\frac{y}{g_{0}}\right)\left(F_{1 x}+F_{2 x}\right)
\end{gathered}
$$

Where $\alpha$ is geometric coupling. Therefore, application of Newton's second law of motion yields rotor dynamics equations:

$$
\begin{gathered}
m \ddot{x}=F_{x}+F_{a x}+m u \omega^{2} \cos \omega t \\
m \ddot{y}=F_{y}+F_{a y}+m u \omega^{2} \sin \omega t-m g
\end{gathered}
$$

where $m$ is half of the rotor mass, $u$ is eccentricity, $\omega$ is the rotor angular speed, $F_{\text {ax }}$ and $F_{\text {ay }}$ arethe auxiliary contact forces in $X$ and $Y$ directions respectively. Unbalance mass is assumed in the middle of rotor. $F_{\text {ax }}$ and $F_{\text {ay }}$ are calculated as follows:

$$
\begin{aligned}
& F_{a x}=-F_{n} \cos \psi+F_{t} \sin \psi \\
& F_{a x}=-F_{n} \sin \psi-F_{t} \cos \psi
\end{aligned}
$$

Where $F_{n}$ and $F_{t}$ are normal and tangential contact forces respectively. $\psi$ is the rotor relative position vector angle with $\mathrm{x}$ axis. These contact parameters can be calculated as follows:

In contact free mode penetration depth (e) is not positive $\mathrm{F} n=F_{t}=0$ for $e<=0$

In contact mode penetration depth is positive.

$$
\begin{gathered}
\mathrm{F}_{\mathrm{n}}=\mathrm{ke} \quad \text { for } \mathrm{e}>0 \\
\mathrm{~F}_{\mathrm{t}}=\mu \mathrm{ke} \quad \text { for } \mathrm{e}>0 \\
\mathrm{e}=\left(\mathrm{x}^{2}+\mathrm{y}^{2}\right)^{1 / 2}-0.5 \mathrm{~g}_{0} \text { and } \psi=\arctan (\mathrm{y} / \mathrm{x})
\end{gathered}
$$

where $k$ is contact stiffness and $\mu$ is sliding friction coefficient. The stiffness is assumed linear. In other words Hertzian's Theory is used to calculate normal forces. Friction forces are considered to be proportional to the 
normal forces (Coulomb friction).Dividing both side of the dynamic Equation (6) by $m \omega_{n}{ }^{2} g_{0}$ and substituting $\bar{x}=\frac{x}{g_{0}} ; \quad \bar{y}=\frac{y}{g_{0}} ; \quad U=\frac{u}{g_{0}} ; \quad \Omega=\frac{\omega}{\omega_{n}} ; \quad \tau=\omega_{n} t ; \quad w=\frac{g}{g_{0} \omega_{n}^{2}}$ then on dimensional forms of theequations will be as follows:

$$
\begin{aligned}
& \ddot{\bar{x}}=\bar{F}_{x}+\bar{F}_{a x}+U \Omega^{2} \cos \Omega \tau \\
& \ddot{\bar{y}}=\bar{F}_{y}+\bar{F}_{a y}+U \Omega^{2} \sin \Omega \tau-w
\end{aligned}
$$

Where:

$$
\bar{F}_{j}=F_{j} / m \omega_{n}^{2} g_{0} ; \bar{F}_{a j}=F_{a j} / m \omega_{n}^{2} g_{0}, \mathrm{j}=\mathrm{x}, \mathrm{y}
$$

Dividing contact forces in Equation (8) by $m \omega_{n}{ }^{2} g_{0}$ and defining non dimensional contact stiffness and penetration depth as $K=k / m \omega_{n}{ }^{2}$ and $\varepsilon=e / g_{0}$ respectively equation (6) in non dimensional form is as follows:

$$
\begin{aligned}
& \bar{F}_{a x}=\mathrm{F}_{\mathrm{ax}} / \mathrm{m} \omega_{\mathrm{n}}{ }^{2} \mathrm{~g}_{0}=-\mathrm{K} \varepsilon \cos \psi+\mu \mathrm{K} \varepsilon \sin \psi \\
& \bar{F}_{a y}=\mathrm{F}_{\mathrm{ax}} / \mathrm{m} \omega_{\mathrm{n}}{ }^{2} \mathrm{~g}_{0}=-\mathrm{K} \varepsilon \sin \psi-\mu \mathrm{K} \varepsilon \cos \psi
\end{aligned}
$$

Time varying stiffnessin non dimensional form is as follows:

$$
\overline{K_{p}}=\overline{P_{1}}+\overline{P_{2}} \cos \Omega \tau
$$

Where

$$
\overline{P_{j}}=\frac{P_{j} g_{0}}{i_{b}} ; \mathrm{j}=1,2 \quad \overline{K_{p}}=\frac{K_{p} g_{0}}{i_{b}}
$$

Where $P_{1}$ and $P_{2}$ are constant coefficients. Substituting $K_{p}$ in Equation (3) and expanding Equation (9) about equilibrium position $(\mathrm{x}, \mathrm{y})=(0,0)$ using Taylor series leads to Mathieu's equations. The stability of the system with the selected coefficients confirmed for contact free mod. As aresult time variable stiffness decrease rotor displacement in contact free mode with smooth rotor whirl orbit. This prevents rotor contact with the auxiliary bearing. This causes more synchronous responses.

\section{Resuts and Discussions}

Simulation is carried out for a rigid rotor supported by two magnetic bearings and two auxiliary bearings. Two modes of motion are considered contact mode and contact free mode. The results are obtained for systems with linear PD controller and for systems with time varying stiffness. They are compared to each others for similar cases. System dynamics behaviorare depicted in periodic, quasi periodic and chaotic forms. Figures 3a-c illustrate bifurcation diagram for magnetic bearing with auxiliary bearing using linear PD controller. There is synchronous response for $\Omega<0.42$. It is period- 2 when $0.42<\Omega<0.46$ and period-5 when $0.41<\Omega<0.56$. By increasing speed to $\Omega=1.7$ the rotor response will be synchronous. This is confirmed by Figures 4,5 and 6 . Figure 4 is rotor whirl orbit and Figure 5 is system power spectrum. All these three figures reveal 5 different frequency for $\Omega=0.55$. Figure 6 is Poincare map. Whentime varying stiffness is used the rotor response is synchronous in speed range $\Omega<0.7$ (Figure 7).

The response will be non synchronous by increasing speed. It is period-3 when $\Omega>1.7$.

This illustrates a delay in bifurcation onset. The delay is around 0.28 which is significant. The rotor whirl orbit and power spectrum signal for $\Omega=0.55$ reveal one dominant frequency which confirm the synchronous response in this region (Figures 8-9).

It is observed from the results that at lower speed in contact free mode time varying stiffnessachieve better rotor whirl orbit and therefore smaller rotor displacement. Smaller rotor displacement prevents contact and therefore continues motion in contact free mode. This causesa delay in bifurcation onset. This means delay in asynchronous and chaos motion. This result in a better system dynamic performance. 


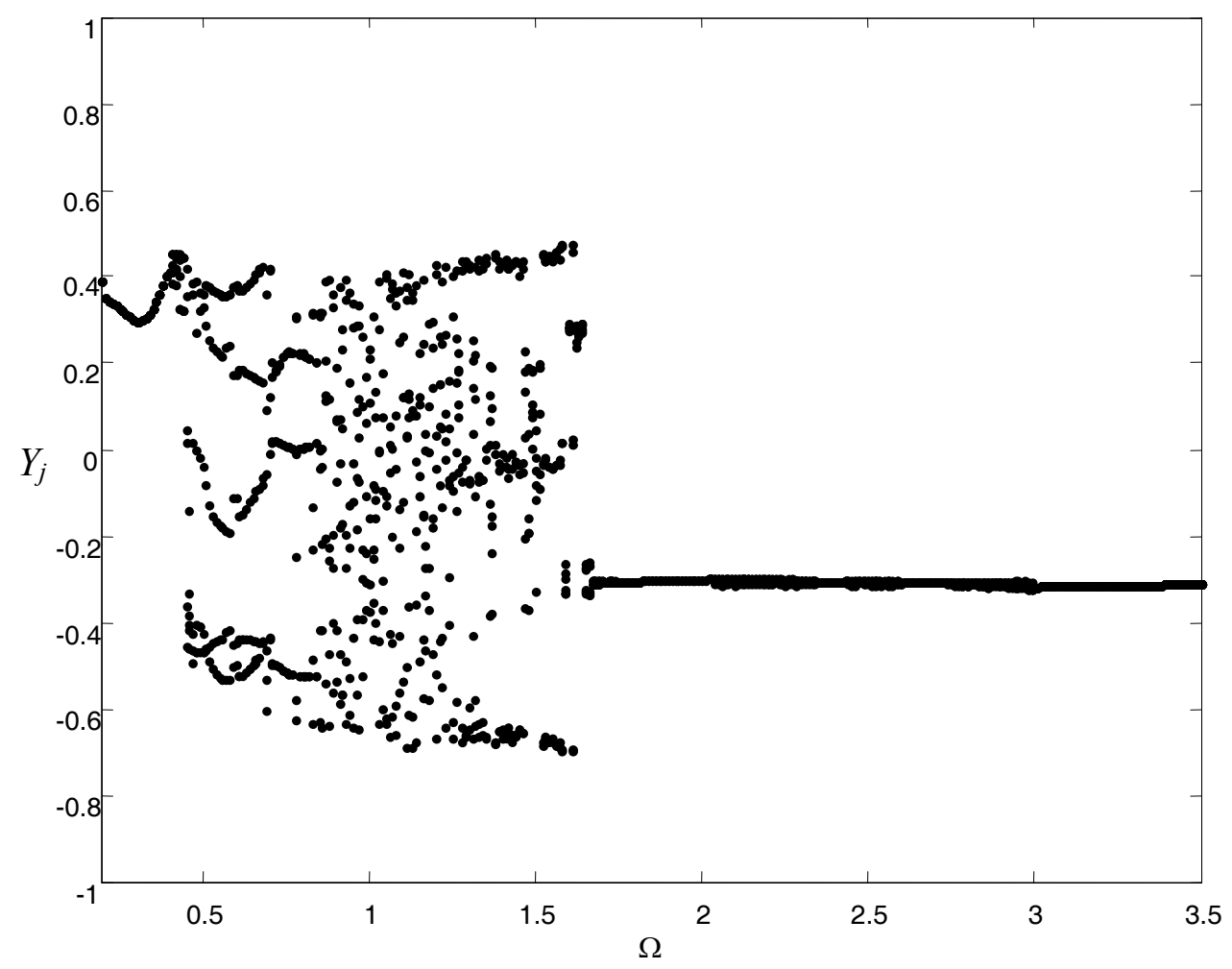

Figure 3a. Bifurcation diagram for auxiliary bearing using $\mathrm{PD}$ controller $\mathrm{U}=0.2, \mathrm{~K}=0.2, \mu=0.3, \mathrm{~W}=0$

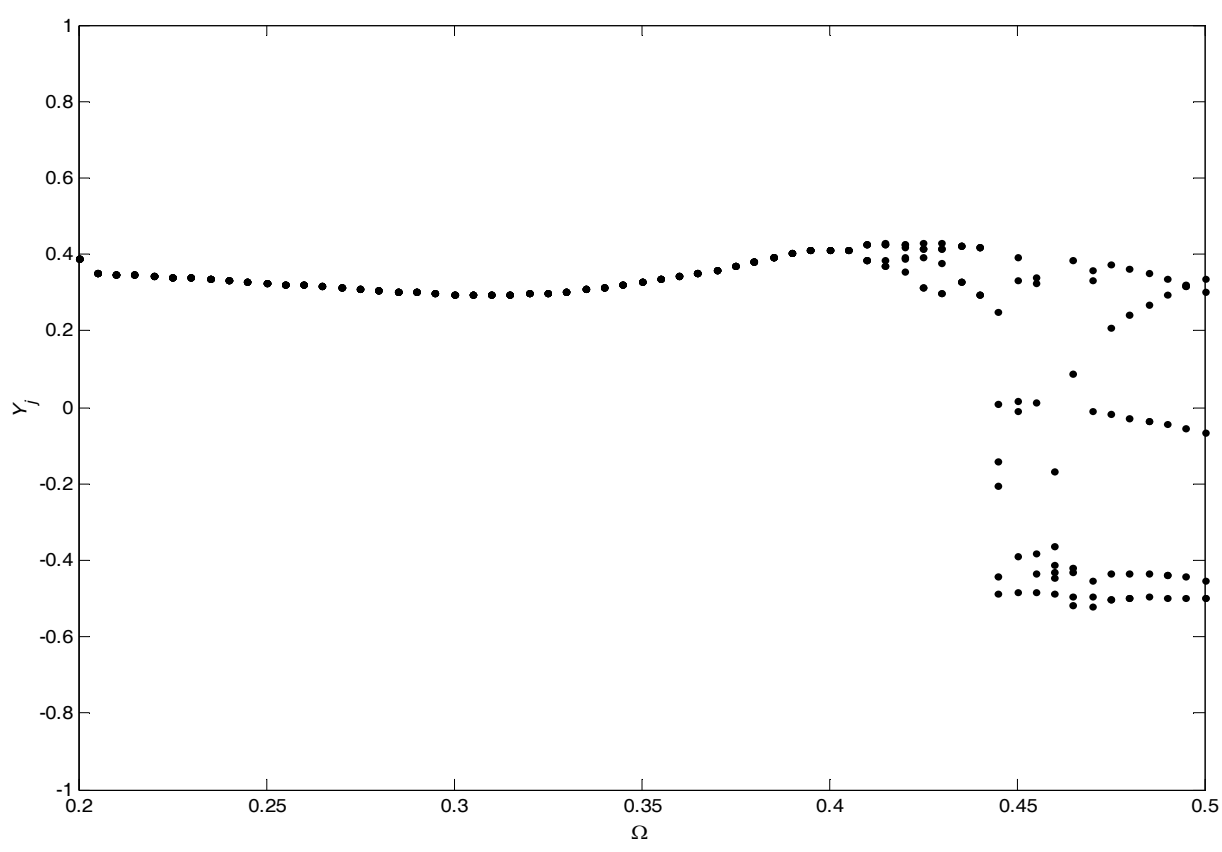

Figure $3 b$. Bifurcation diagram for auxiliary bearing using $P D$ controller $U=0.2, K=0.2, \mu=0.3, W=0$ 


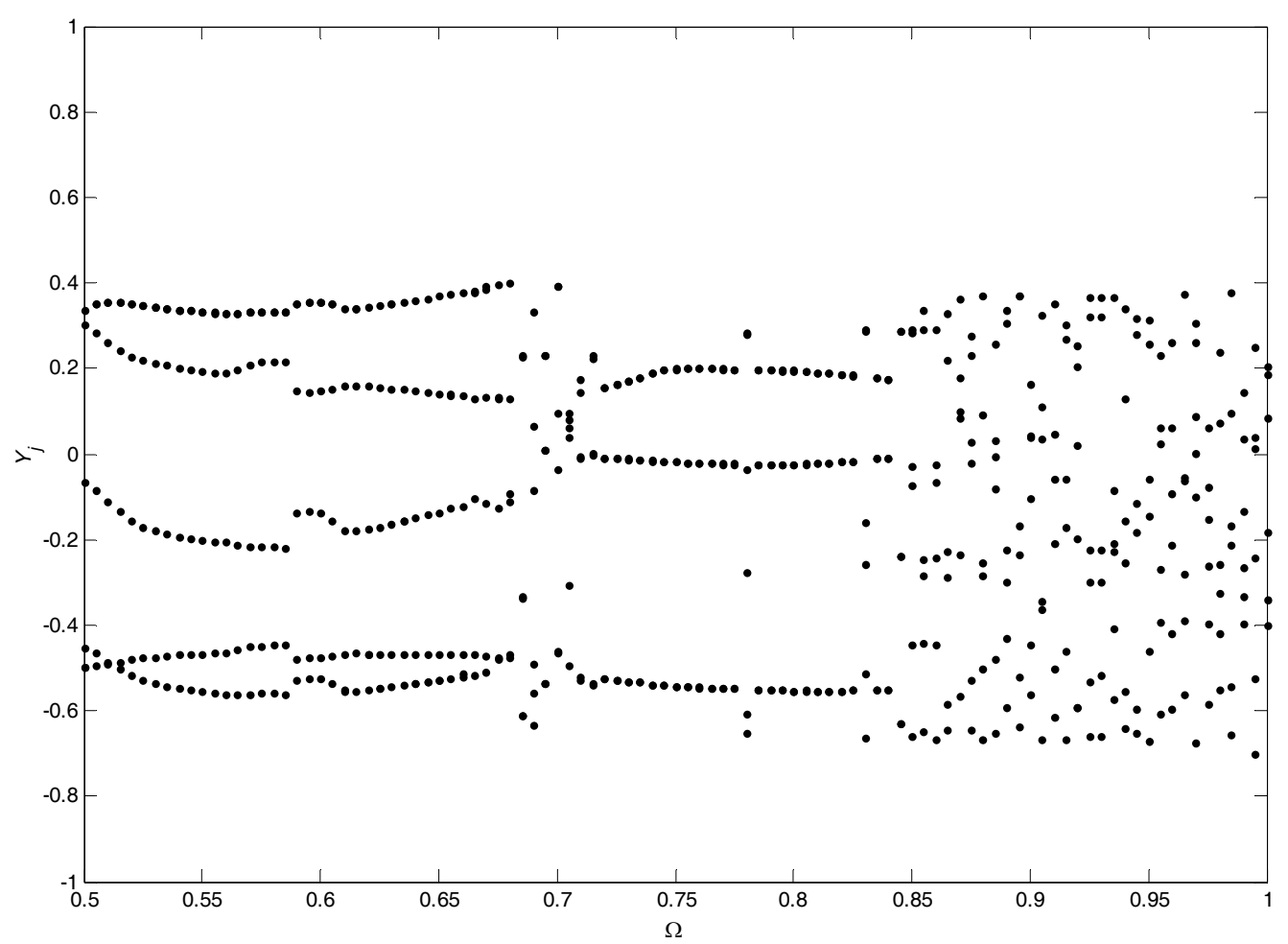

Figure $3 c$. Bifurcation diagram for auxiliary bearing using $P D$ controller $U=0.2, K=0.2, \mu=0.3, W=0$

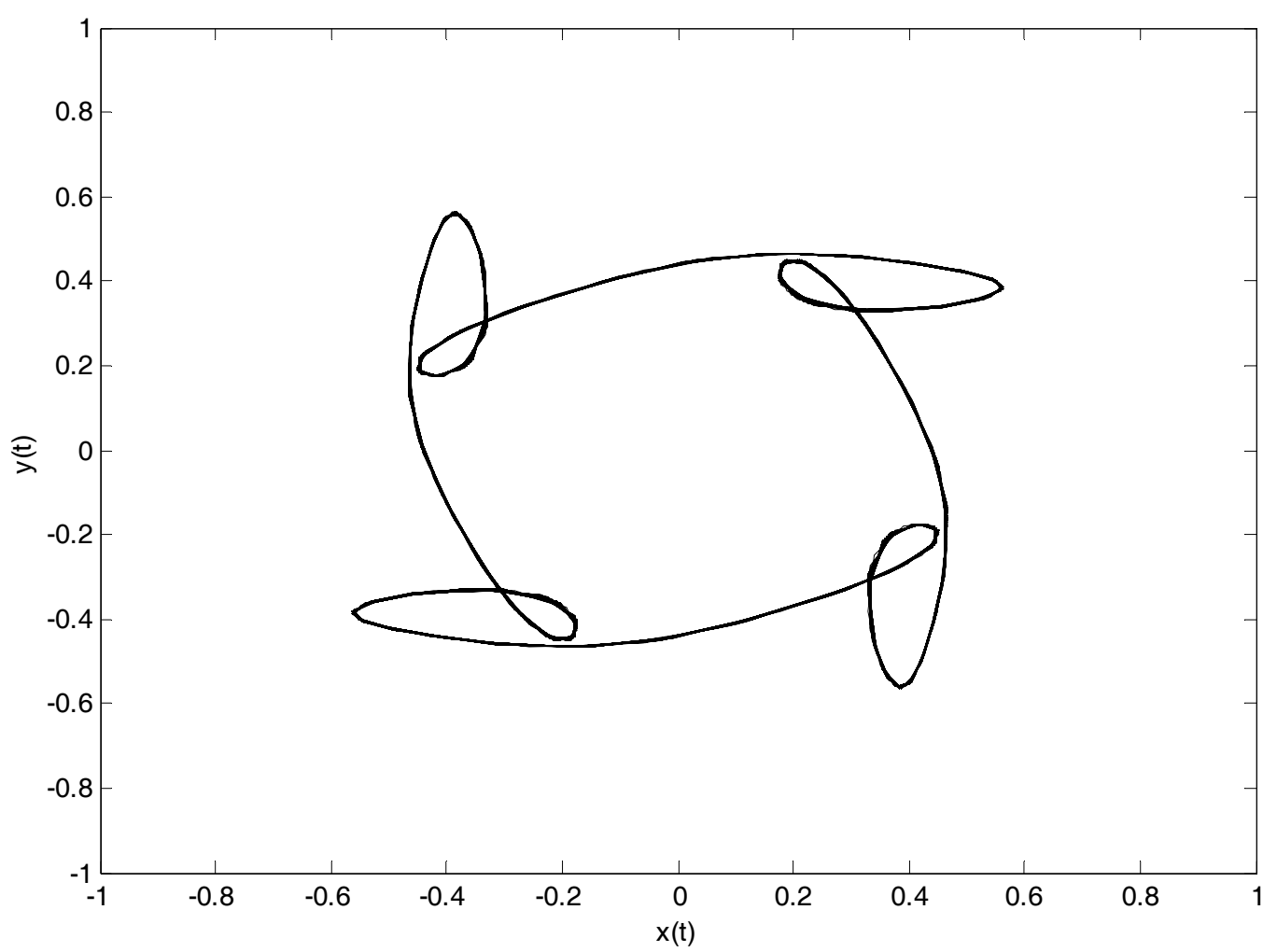

Figure 4. Rotor whirl orbit for auxiliary bearing using PD controller $\mathrm{U}=0.2, \Omega=0.55, \mu=0.3 \mathrm{~K}=0.2, \mathrm{~W}=0$ 


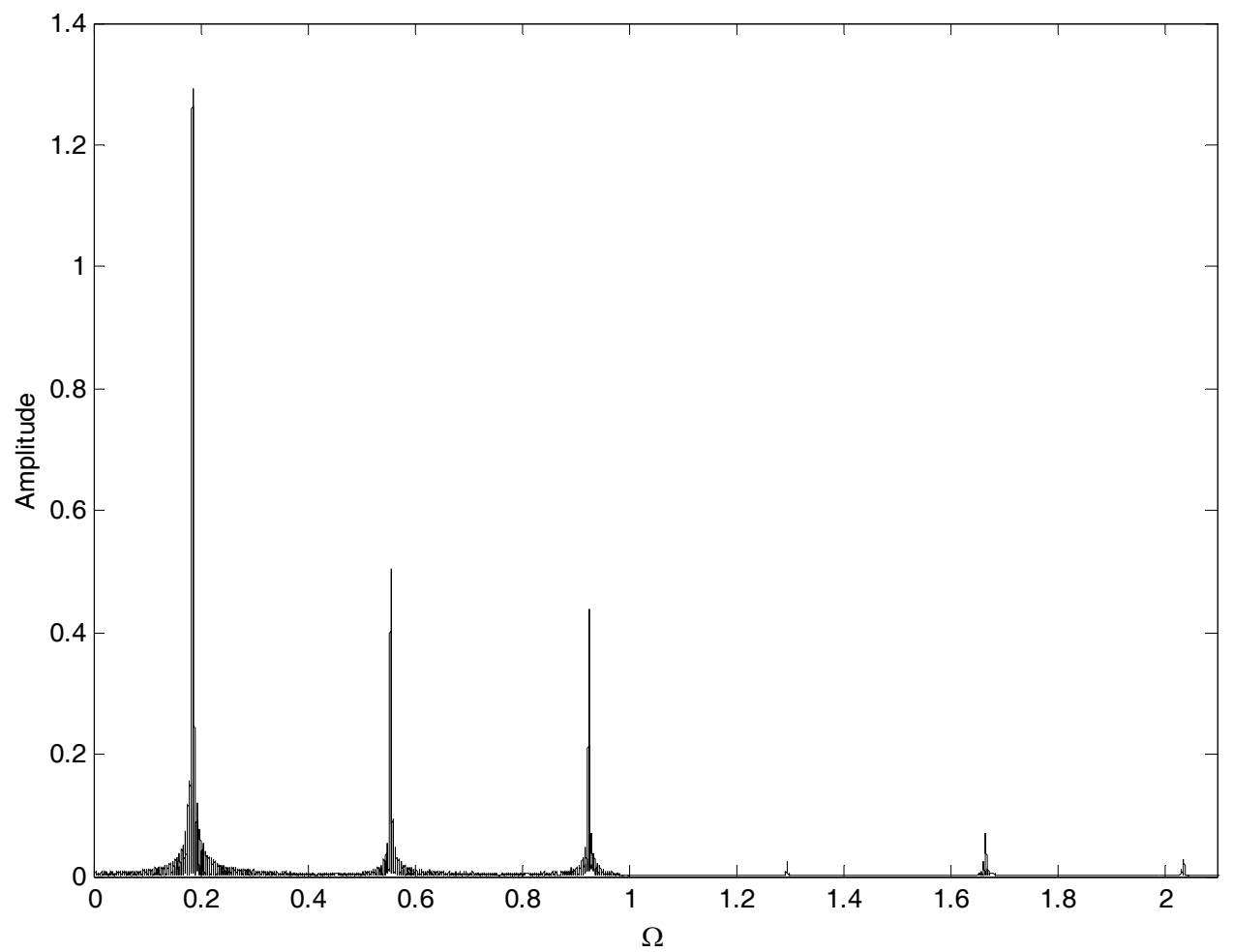

Figure 5. Power spectrum for auxiliary bearing using PD controller $U=0.2, \Omega=0.55, \mu=0.3 \mathrm{~K}=0.2, \mathrm{~W}=0$

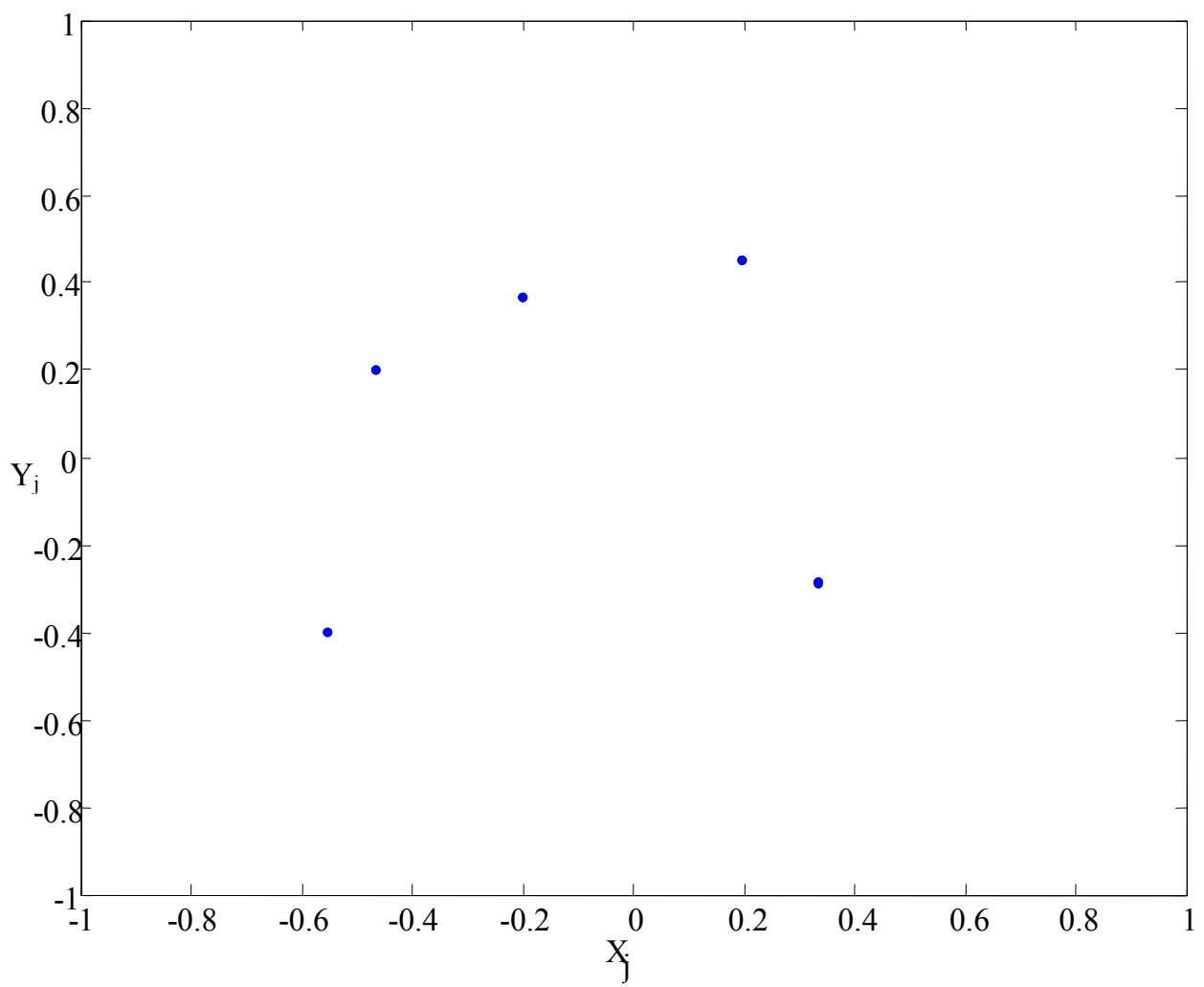

Figure 6. Poincare map for auxiliary bearing using PD controller $U=0.2, \Omega=0.55, \mu=0.3 \mathrm{~K}=0.2, \mathrm{~W}=0$ 


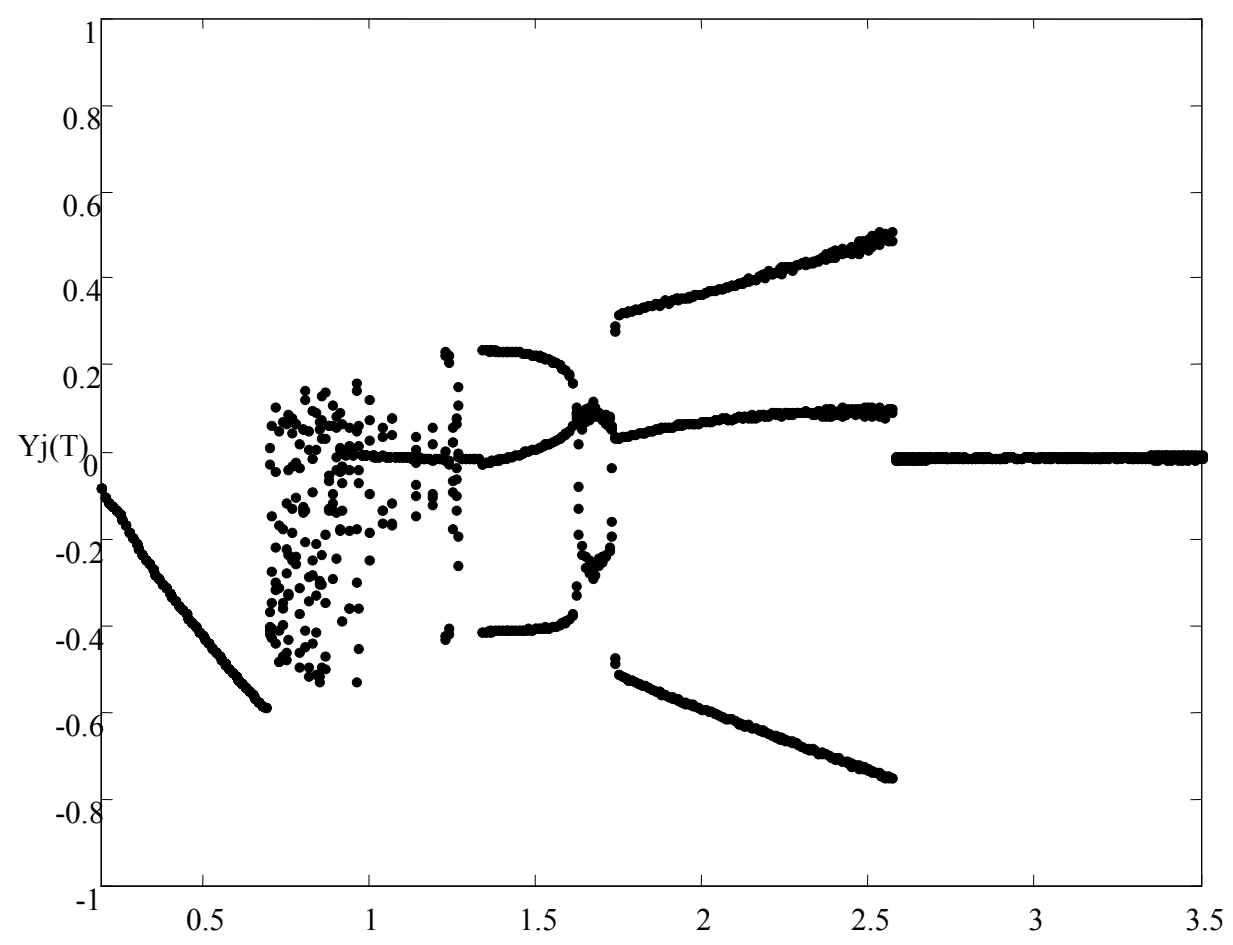

Figure 7. Bifurcation diagram for auxiliary bearing using time varying stiffness $U=0.2, K=0.2, \mu=0.3, W=0$

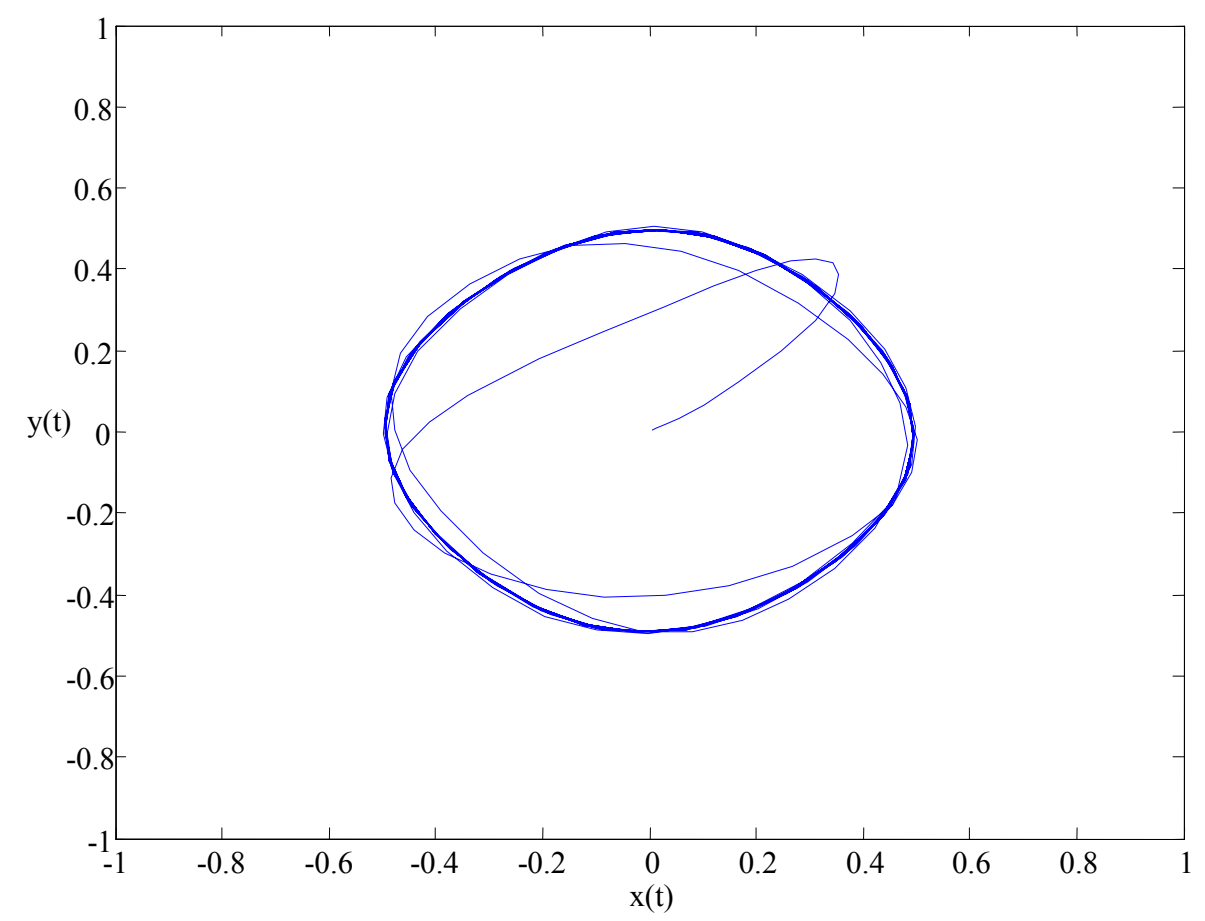

Figure 8. Rotor whirl orbit for auxiliary bearing using time varying stiffness

$$
\mathrm{U}=0.2, \Omega=0.55, \mu=0.3 \mathrm{~K}=0.2, \mathrm{~W}=0
$$




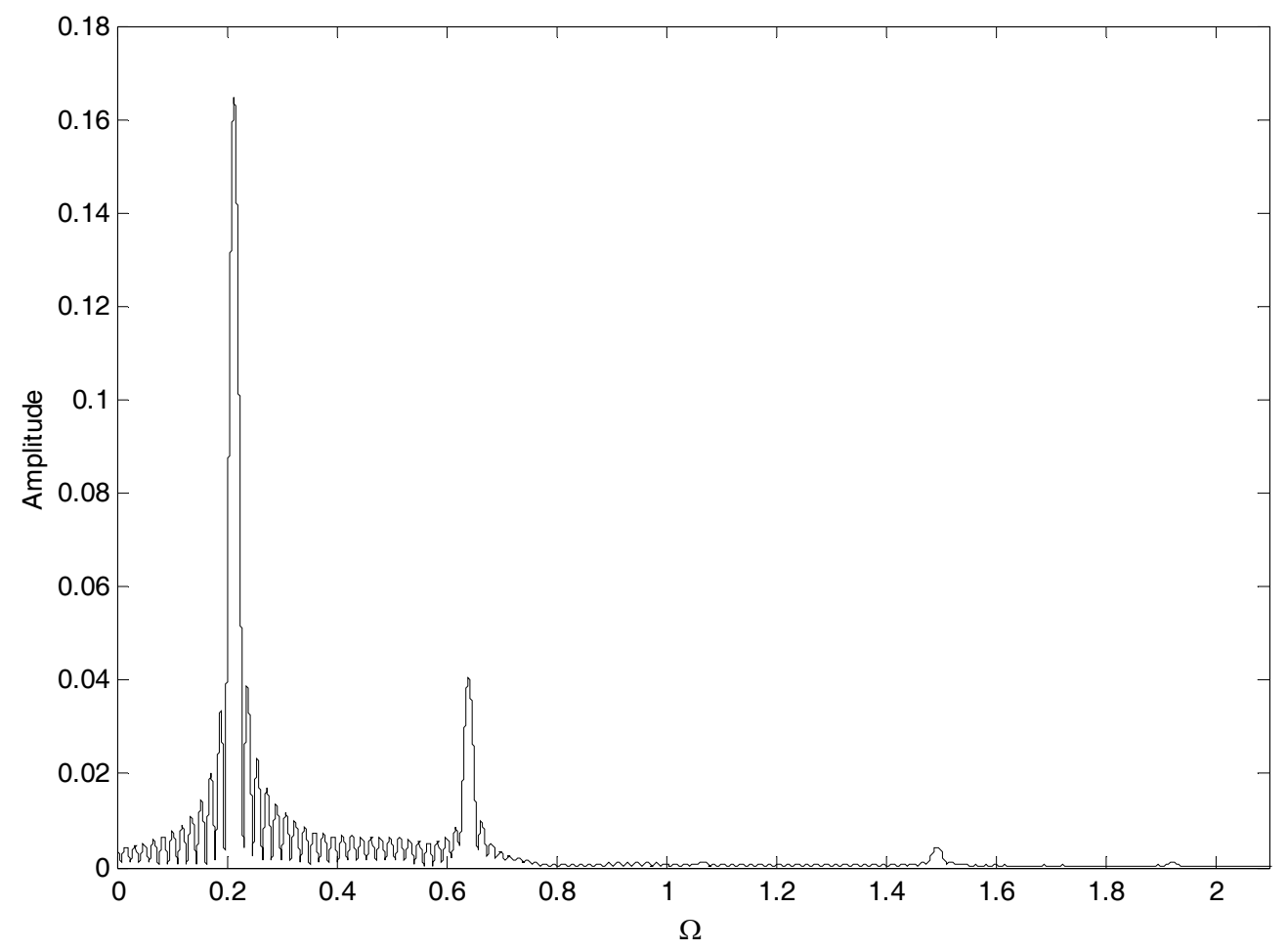

Figure 9. Power spectrum for auxiliary bearing using time varying stiffness

$$
\mathrm{U}=0.2, \Omega=0.55, \mu=0.3 \mathrm{~K}=0.2, \mathrm{~W}=0
$$

\section{Conclusion}

In magnetic bearing system with auxiliary bearing time varying stiffness modify the rotor response at low speed. The rotating speed range with synchronous response increases. This is revealed by delaying bifurcation onset, since non-synchronous vibrations resulting stress fluctuation and failure. When unbalance is high and machinery speed is less than first natural frequency $(\Omega<1)$ time varying stiffness is preferable.

\section{References}

Cade Iain, S. M., Sahinkaya, N., Burrows, Clifford, R., \& Keough Patrick, S. (2008). On the design of an active magnetic bearing systems. $11^{\text {th }}$ International symposium on Magnetic Bearings, August 26-29, Nara, Japan.

Ghazavi, M. R., \& Sun, Q. (2012). Nonlinear Dynamics Behaviors in Magnetic Bearing Systems. (In Process)

Inayat-Hussain, J. I. (2010). Nonlinear dynamics of a magnetically supported rigid rotor inauxiliary bearings. Mechanism and Machine Theory, 45, 1651-1667. http://dx.doi.org/10.1016/j.mechmachtheory.2010.06.006

Inayat-Hussain, J. I. (2011). Bifurcations in the response of a rigid rotor supportedby load sharing between magnetic and auxiliary bearings. Meccanica, 46(6), 1341-1351. http://dx.doi.org/10.1007/s11012-010-9395-8

Jang, M. J., \& Chen, C. K. (2001). Bifurcation analysis in flexible rotor supported by active magnetic bearings. Int. J. Bifurcat. Chaos, 11, 2163-2178. http://dx.doi.org/10.1142/S0218127401003437

Wang, X., \& Noah, S. (1998). Nonlinear dynamics of a magnetically supported rotor on safety auxiliary bearings. ASME J. Vib. Acoust., 120, 596-606. http://dx.doi.org/10.1115/1.2893869

Zeng, S. (2002). Motion of AMB rotor in backup bearings. ASME J. Vib. Acoust., 124, 460-464. http://dx.doi.org/10.1115/1.1476382 